\title{
Choquet and Shilov Boundaries, Peak Sets, and Peak Points for Real Banach Function Algebras
}

\author{
Davood Alimohammadi ${ }^{1}$ and Taher Ghasemi Honary ${ }^{2}$ \\ ${ }^{1}$ Department of Mathematics, Faculty of Science, Arak University, Arak 38156-8-8349, Iran \\ ${ }^{2}$ Faculty of Mathematical Sciences and Computer, Kharazmi University (Tarbiat Moallem University), 50 Taleghani Avenue, \\ Tehran 15618-36314, Iran \\ Correspondence should be addressed to Davood Alimohammadi; d-alimohammadi@araku.ac.ir
}

Received 5 December 2012; Accepted 1 March 2013

Academic Editor: Dachun Yang

Copyright (c) 2013 D. Alimohammadi and T. G. Honary. This is an open access article distributed under the Creative Commons Attribution License, which permits unrestricted use, distribution, and reproduction in any medium, provided the original work is properly cited.

Let $X$ be a compact Hausdorff space and let $\tau$ be a topological involution on $X$. In 1988, Kulkarni and Arundhathi studied Choquet and Shilov boundaries for real uniform function algebras on $(X, \tau)$. Then in 2000, Kulkarni and Limaye studied the concept of boundaries and Choquet sets for uniformly closed real subspaces and subalgebras of $C(X, \tau)$ or $C(X)$. In 1971, Dales obtained some properties of peak sets and $p$-sets for complex Banach function algebras on $X$. Later in 1990, Arundhathi presented some results on peak sets for real uniform function algebras on $(X, \tau)$. In this paper, while we present a brief account of the work of others, we extend some of their results, either to real subspaces of $C(X, \tau)$ or to real Banach function algebras on $(X, \tau)$.

\section{Introduction and Preliminaries}

Let $\mathbb{F}$ denote either $\mathbb{R}$ or $\mathbb{C}$. We always assume that $X$ is a compact Hausdorff space. We denote by $C_{\mathbb{F}}(X)$ the Banach algebra of all continuous functions from $X$ into $\mathbb{F}$, with the uniform norm

$$
\|f\|_{X}=\sup \{|f(x)|: x \in X\} \quad\left(f \in C_{\mathbb{F}}(X)\right) .
$$

However, we always write $C(X)$ instead of $C_{\mathbb{C}}(X)$ and we denote the uniform closure of $A$ by $\bar{A}$, whenever $A$ is a subset of $C(X)$.

Let $A$ be a real or complex subspace of $C(X)$. A nonempty subset $S$ of $X$ is called a boundary (Choquet set, resp.) for $A$ (with respect to $X$ ), if for each $f \in A$ the function $|f|(\operatorname{Re} f$, resp.) assumes its maximum on $X$ at some $x \in S$. Note that every closed boundary for $A$ is a closed Choquet set for $A$ [1, Lemma 1.1] and if $A$ is closed under the complex scalar multiplication, then every Choquet set for $A$ is a boundary for $A[1$, Section 1$]$. We denote by $\Gamma(A, X)$ the intersection of all closed boundaries for $A$. If $\Gamma(A, X)$ is a boundary for $A$, it is called the Shilov boundary for $A$.
Let $A$ be a subspace of $C(X)$ over $\mathbb{F}$ containing 1 . We denote by $K_{\mathbb{F}}(A)$ the set of all $\varphi \in A^{*}$ for which $\|\varphi\|=$ $\varphi(1)=1$, where $A^{*}$ is the dual space of the normed space $\left(A,\|\cdot\|_{X}\right)$ over $\mathbb{F}$. In fact, the elements of $A^{*}$ are $\mathbb{F}$-valued linear functionals on $A$ over $\mathbb{F}$. For each $x \in X$ the map $e_{x}: A \rightarrow \mathbb{C}$, defined by $e_{x}(f)=f(x)$, is a linear mapping over $\mathbb{F}$, which is called the evaluation map on $A$ at $x$. Clearly $e_{x} \in K_{\mathbb{C}}(A)$ whenever $\mathbb{F}=\mathbb{C}$ and $\operatorname{Re} e_{x} \in K_{\mathbb{R}}(A)$ whenever $\mathbb{F}=\mathbb{R}$.

Let $B$ be a complex subspace of $C(X)$ containing 1 . A representing measure for $\varphi \in B^{*}$ is a complex regular Borel measure $\mu$ on $X$ such that $\varphi(f)=\int_{X} f d \mu$ for all $f \in B$. It is known that every $\varphi \in K_{\mathbb{C}}(B)$ has a representing measure and every representing measure for such $\varphi$ is a probability measure [2, Section 2.1]. If $x \in X$, then $\delta_{x}$, the point mass measure at $x$, is a representing measure for $e_{x}$. We denote by $C h(B, X)$ the set of all $x \in X$ for which $\delta_{x}$ is the only representing measure for $e_{x}$. If $C h(B, X)$ is a boundary for $B$, it is called the Choquet boundary for $B$.

Let $A$ be a real subspace of $C(X)$ containing 1 . A real part representing measure for $\psi \in A^{*}$ is a regular Borel measure $\mu$ on $X$ such that $\psi(f)=\int_{X} \operatorname{Re} f d \mu$ for all $f \in A$. It is 
wellknown that every $\psi \in K_{\mathbb{R}}(A)$ has a real part representing measure [1, Theorem 1.5]. If $x \in X$, then $\delta_{x}$ is a real part representing measure for $\operatorname{Re} e_{x}$. We denote by $\operatorname{Ch}(A, X)$ the set of all $x \in X$ for which $\delta_{x}$ is the only real part representing measure for $\operatorname{Re} e_{x}$. If $C h(A, X)$ is a boundary for $A$, it is called the Choquet boundary for $A$.

Let $E$ be a nonempty set. A self-map $\tau: E \rightarrow E$ is called an involution on $E$ if $\tau(\tau(x))=x$ for all $x \in E$. We denote by $\operatorname{Fix}(\tau)$ the set of all $x \in X$ for which $\tau(x)=x$. A subset $S$ of $E$ is called $\tau$-invariant if $\tau(S)=S$. A $\tau$-invariant measure on $E$ is a measure $\mu$ on $E$ such that $\mu \circ \tau=\mu$.

An involution $\tau$ on $X$ is called a topological involution on $X$ if $\tau$ is continuous. The map $\sigma: C(X) \rightarrow C(X)$ defined by $\sigma(f)=\bar{f} \circ \tau$ is an algebra involution on $C(X)$, which is called the algebra involution induced by $\tau$ on $C(X)$. We now define

$$
C(X, \tau)=\{f \in C(X): \sigma(f)=f\} .
$$

Then $C(X, \tau)$ is a unital self-adjoint uniformly closed real subalgebra of $C(X)$, which separates the points of $X$ and does not contain the constant function $i$. Moreover, $C(X)=$ $C(X, \tau) \oplus i C(X, \tau)$ and

$$
\max \left\{\|f\|_{X},\|g\|_{X}\right\} \leq\|f+i g\|_{X} \leq 2 \max \left\{\|f\|_{X},\|g\|_{X}\right\}
$$

for all $f, g \in C(X, \tau)$. In fact, the complex Banach algebra $\left(C(X),\|\cdot\|_{X}\right)$ can be regarded as the complexification of the real Banach algebra $\left(C(X, \tau),\|\cdot\|_{X}\right)$. Note that $C(X, \tau)=$ $C_{\mathbb{R}}(X)$ if and only if $\tau$ is the identity map on $X$. Hence the class real Banach algebras of continuous complex-valued functions $C(X, \tau)$ are, in fact, larger than the class of real Banach algebras of continuous real-valued functions $C_{\mathbb{R}}(X)$. This class of continuous functions was defined explicitly by Kulkarni and Limaye in [3].

Let $A$ be a real subspace of $C(X, \tau)$ containing 1 . We denote by $\Gamma(A, X, \tau)$ the intersection of all $\tau$-invariant closed boundaries for $A$. If $\Gamma(A, X, \tau)$ is a boundary for $A$, we call it the Shilov boundary for $A$ with respect to $(X, \tau)$. For each $x \in X$, the measure $(1 / 2)\left(\delta_{x}+\delta_{\tau(x)}\right)$ is a positive regular Borel measure on $X$, which is denoted by $m_{x}$. If $x \in X$, then $m_{x}$ is a $\tau$-invariant real part representing measure for $\operatorname{Re} e_{x}$. We denote by $C h(A, X, \tau)$ the set of all $x \in X$ for which $m_{x}$ is the only $\tau$-invariant real part representing measure for $\operatorname{Re} e_{x}$. If $C h(A, X, \tau)$ is a boundary for $A$, we call it the Choquet boundary for $A$ with respect to $(X, \tau)$.

For a topological involution $\tau$ on $X$ let $X / \tau=\{\{x, \tau(x)\}$ : $x \in X\}$. For a subset $A$ of $C(X, \tau)$ we say that $\operatorname{Re} A$ separates the points of $X / \tau$ if for each $x \in X$ and $y \in X \backslash\{x, \tau(x)\}$, there exists a function $f$ in $A$ such that $\operatorname{Re} f(x) \neq \operatorname{Re} f(y)$. It is interesting to note that if $A$ is a real subalgebra of $C(X, \tau)$ which separates the points of $X$ and contains 1, then $\operatorname{Re} A$ separates the points of $X / \tau$ [4, Lemma 1.3.9].

Let $A$ be a real or complex subalgebra of $C(X)$ and let $S$ be a nonempty subset of $X$. Then $S$ is called a peak set for $A$ if there exists $f \in A$ such that $S=\{x \in X: f(x)=1\}$ and $|f(y)|<1$ for all $y \in X \backslash S$. We say that $S$ is a $p$-set (a peak set in the weak sense or a weak peak set) for $A$ if $S$ is the intersection of some collection of peak sets for $A$. If the peak set or $p$-set $S$ for $A$ is a singleton $\{x\}$, then we call $x$ a peak point or p-point for $A$. The set of all peak points, or $p$-points, for $A$ is denoted by $S_{0}(A, X)$, or $S(A, X)$, respectively. Note that $S_{0}(A, X)$ is the intersection of all boundaries for $A$.

If $A$ is a real subalgebra of $C(X, \tau)$, then every $p$-set and hence every peak set for $A$ are $\tau$-invariant. Hence $S_{0}(A, X) \subseteq$ $S(A, X) \subseteq \operatorname{Fix}(\tau)$. Moreover, $S_{0}(A, X)$ is contained in every $\tau$-invariant boundary for $A$.

Definition 1. Let $A$ be a real subspace of $C(X, \tau)$. The point $x \in$ $X$ is a $\tau$-peak point ( $\tau$-p-point, resp.) for $A$ if the set $\{x, \tau(x)\}$ is a peak set (a $p$-set, resp.) for $A$. We define

$$
\begin{gathered}
T_{0}(A, X, \tau)=\{x \in X: x \text { is a } \tau \text {-peak point for } A\}, \\
T(A, X, \tau)=\{x \in X: x \text { is a } \tau \text {-p-point for } A\} .
\end{gathered}
$$

It is easy to see that $T_{0}(A, X, \tau)$ is the intersection of all $\tau$-invariant boundaries for $A$.

Definition 2. A complex Banach function algebra on $X$ is a complex subalgebra of $C(X)$ which contains 1 , separates the points of $X$, and it is a unital Banach algebra under an algebra norm $\|\cdot\|$. If the norm of a complex Banach function algebra on $X$ is the uniform norm on $X$, then it is called a complex uniform function algebra on $X$.

Clearly, $C(X)$ is a complex uniform function algebra on $X$.

Let $B$ be a unital commutative Banach algebra over $\mathbb{F}$. A character of $B$ is a nonzero homomorphism $\varphi: B \rightarrow \mathbb{C}$, where $\mathbb{C}$ is regarded as an algebra over $\mathbb{F}$. We denote by $M_{B}$ the set of all characters of $B$. It is known that $M_{B}$, with the Gelfand topology, is a compact Hausdorff space (see [5, Chapter 11] and [4, Chapter 1]).

Definition 3. A real Banach function algebra on $(X, \tau)$ is a real subalgebra of $C(X, \tau)$ which contains 1 , separates the points of $X$, and it is a real unital Banach algebra under an algebra norm $\|$. \|. If the norm of a real Banach function algebra on $(X, \tau)$ is the uniform norm on $X$, then it is called a real uniform function algebra on $(X, \tau)$.

Clearly, $C(X, \tau)$ is a real uniform function algebra on $(X, \tau)$.

Since the class of real uniform (or Banach) function algebras on $(X, \tau)$ is larger than the class of complex uniform (or Banach) function algebras, it is quite natural to ask which properties of a complex algebra can be extended to the corresponding real algebra.

Let $A$ be a real (complex, resp.) Banach function algebra on $(X, \tau)$ (on $X$, resp.). The evaluation character $e_{x}$ is an element of $M_{A}$ for all $x \in X$. We call $A$ to be natural if every $\varphi \in M_{A}$ is given by an evaluation character $e_{x}$ at some $x \in X$.

The concept of the Shilov boundary for a real Banach algebra was studied by Ingelstam, Limaye, and Simha in [68]. The concept of Choquet and Shilov boundaries for real uniform function algebras was first studied by Kulkarni and Arundhathi in [9]. Later Kulkarni and Limaye introduced the notions of Choquet sets and boundaries for real subspaces of $C(X)$ and $C(X, \tau)$ in [1] and obtained interesting results on Choquet and Shilov boundaries for uniformly closed real subalgebras of $C(X)$ or $C(X, \tau)$. They also obtained relations between $C h(A, X, \tau)$ and $C h(B, X)(\Gamma(A, X, \tau)$ and $\Gamma(B, X)$, 
resp.), when $A$ is a real uniform function algebra on $(X, \tau)$ and $B=\{f+i g: f, g \in A\}$ is the complexification of $A$.

In Section 2, we first show that $\operatorname{Ch}(A, X)=\operatorname{Ch}(\bar{A}, X)$ and $\Gamma(A, X)=\Gamma(\bar{A}, X)$ and by applying these equalities we extend some results due to Kulkarni, Limaye, and Arundhathi, by omitting the uniformly closed condition on $A$.

The concept of peak points and $p$-points for real uniform function algebras was studied by Kulkarni and Arundhathi in $[9,10]$. Also Dales has obtained many results on the boundaries and peak sets for complex Banach function algebras in $[11,12]$. In Sections 3 and 4, we extend some of their results to real Banach function algebras.

\section{Choquet and Shilov Boundaries}

We begin this section by recalling some well-known results.

Theorem 4 (see [4, Theorem 4.3.3 and Corollary 4.3.4]). Let $B$ be a complex subspace of $C(X)$ containing 1 and separating the points of $X$. Then

(i) $\varphi \in K_{\mathbb{C}}(B)$ is an extreme point of $K_{\mathbb{C}}(B)$ if and only if there exists $x \in C h(B, X)$ such that $\varphi=e_{x}$. In particular, $C h(B, X)$ is nonempty.

(ii) $\operatorname{Ch}(B, X)$ is the set of all $x \in X$ such that $e_{x}$ is an extreme point of $K_{\mathbb{C}}(B)$.

(iii) $C h(B, X)$ is a Choquet set for $B$ and, in particular, $a$ boundary for $B$.

(iv) The closure of $C h(B, X)$ is equal to $\Gamma(B, X)$.

Theorem 5 (see [1, Corollary 1.8]). Let A be a real subspace of $C(X)$. Then the closure of $C h(A, X)$ is contained in every closed Choquet set as well as every closed boundary for $A$. In particular, $C h(A, X) \subseteq \Gamma(A, X)$.

Theorem 6 (see [1, Lemma 2.1 and Theorem 2.2]). Let $A$ be a real subspace of $C(X)$ containing 1 and $\operatorname{Re} A$ separates the points of $X$. Then

(i) The element $\varphi \in K_{\mathbb{R}}(A)$ is an extreme point of $K_{\mathbb{R}}(A)$ if and only if $\varphi=\operatorname{Re} e_{x}$ for some $x \in \operatorname{Ch}(A, X)$. In particular, $C h(A, X)$ is nonempty.

(ii) $\operatorname{Ch}(A, X)$ is the set of all $x \in X$ such that $\operatorname{Re} e_{x}$ is an extreme point of $K_{\mathbb{R}}(A)$.

(iii) $C h(A, X)$ is a Choquet set for $A$.

(iv) The closure of $\operatorname{Ch}(A, X)$ is the smallest closed Choquet set for $A$.

Theorem 7 (see [4, Theorem 4.2.2((a), (b))]). Let $A$ be a real subspace of $C(X, \tau)$ containing 1 .

(i) If $S$ is a $\tau$-invariant boundary for $A$, then the closure of $S$ is a $\tau$-invariant Choquet set for $A$.

(ii) $\overline{\operatorname{Ch}(A, X, \tau)} \subseteq \Gamma(A, X, \tau)$.

Theorem 8 (see [4, Theorem 4.1.10 and Corollary 4.1.11] and $[1$, Section 4$])$. Let $A$ be a real subspace of $C(X, \tau)$ containing 1 and let $\operatorname{Re} A$ separate the points of $X / \tau$. Then (i) $\varphi \in K_{\mathbb{R}}(A)$ is an extreme point of $K_{\mathbb{R}}(A)$ if and only if there exists $x \in \operatorname{Ch}(A, X, \tau)$ such that $\varphi=\operatorname{Re} e_{x}$. In particular, $C h(A, X, \tau)$ is nonempty.

(ii) $\operatorname{Ch}(A, X, \tau)$ is the set of all $x \in X$ such that $\operatorname{Re} e_{x}$ is an extreme point of $K_{\mathbb{R}}(A)$.

(iii) $\operatorname{Ch}(A, X, \tau)$ is a $\tau$-invariant Choquet set for $A$.

(iv) The closure of $\operatorname{Ch}(A, X, \tau)$ is the smallest $\tau$-invariant Choquet set for $A$.

We now show that $C h(A, X)=\operatorname{Ch}(\bar{A}, X)$ and $\Gamma(A, X)=$ $\Gamma(\bar{A}, X)$, for a real or complex subspace $A$ of $C(X)$, and we then extend some results which have been obtained by Kulkarni, Arundhathi, and Limaye in $[1,9]$.

Theorem 9. (i) Let $A$ be a real subspace of $C(X)$ containing 1. If $\operatorname{Re} A$ separates the points of $X$, then $\operatorname{Ch}(A, X)=\operatorname{Ch}(\bar{A}, X)$.

(ii) Let $A$ be a real subspace of $C(X, \tau)$ containing 1 . If $\operatorname{Re} A$ separates the points of $X / \tau$, then $\operatorname{Ch}(A, X, \tau)=\operatorname{Ch}(\bar{A}, X, \tau)$.

(iii) Let $B$ be a complex subspace of $C(X)$ containing 1 . If $B$ separates the points of $X$, then $\operatorname{Ch}(B, X)=\operatorname{Ch}(\bar{B}, X)$ and $\Gamma(B, X)=\Gamma(\bar{B}, X)$.

Proof. (i) For every $x \in X$, let $e_{x}$ and $\varepsilon_{x}$ be the evaluation maps at $x$ on $A$ and $\bar{A}$, respectively. By Theorem 6(ii), we have

$$
\begin{aligned}
& C h(A, X) \\
& \quad=\left\{x \in X: \operatorname{Re} e_{x} \text { is an extreme point of } K_{\mathbb{R}}(A)\right\}, \\
& \operatorname{Ch}(\bar{A}, X) \\
& \quad=\left\{x \in X: \operatorname{Re} \varepsilon_{x} \text { is an extreme point of } K_{\mathbb{R}}(\bar{A})\right\} .
\end{aligned}
$$

Let $x \in \operatorname{Ch}(A, X)$ and $\operatorname{Re} \varepsilon_{x}=(1-t) \Phi+t \Psi$, where $0<t<1$ and $\Phi, \Psi \in K_{\mathbb{R}}(\bar{A})$. Set $\varphi=\left.\Phi\right|_{A}$ and $\psi=\left.\Psi\right|_{A}$. It is easy to show that $\varphi, \psi \in K_{\mathbb{R}}(A)$ and $\operatorname{Re} e_{x}=(1-t) \varphi+t \psi$ on $A$. Hence $\varphi=\psi=\operatorname{Re} e_{x}$. Let $f$ be an arbitrary element of $\bar{A}$. Then there exists a sequence $\left\{f_{n}\right\}$ in $A$ such that $\lim _{n \rightarrow \infty}\left\|f_{n}-f\right\|_{X}=0$. Since $\Phi \in(\bar{A})^{*}$, we have

$$
\begin{aligned}
\Phi(f) & =\lim _{n \rightarrow \infty} \varphi\left(f_{n}\right) \\
& =\lim _{n \rightarrow \infty} \operatorname{Re}\left(\left(f_{n}(x)\right)\right)=\operatorname{Re} f(x)=\left(\operatorname{Re} \varepsilon_{x}\right)(f) .
\end{aligned}
$$

Hence $\Phi=\operatorname{Re} \varepsilon_{x}$. Similarly, $\Psi=\operatorname{Re} \varepsilon_{x}$. Therefore, $\varepsilon_{x}$ is an extreme point of $K_{\mathbb{R}}(\bar{A})$ and so $x \in \operatorname{Ch}(\bar{A}, X)$.

Conversely, let $x \in \operatorname{Ch}(\bar{A}, X)$ and $\operatorname{Re} e_{x}=(1-t) \varphi+t \psi$, where $0<t<1$ and $\varphi, \psi \in K_{\mathbb{R}}(A)$.

Clearly, if $\eta \in A^{*}$ and $\left\{h_{n}\right\}$ is a sequence in $A$ with $\lim _{n \rightarrow \infty}\left\|h_{n}\right\|_{X}=0$, then $\lim _{n \rightarrow \infty} \eta\left(h_{n}\right)=0$.

Now let $f \in \bar{A}$ and let $\left\{f_{n}\right\}$ be a sequence in $A$ with $\lim _{n \rightarrow \infty}\left\|f_{n}-f\right\|_{X}=0$. We define $\widetilde{\varphi}$ and $\widetilde{\psi}$ on $\bar{A}$ by $\widetilde{\varphi}(f)=$ $\lim _{n \rightarrow \infty} \varphi\left(f_{n}\right)$ and $\widetilde{\psi}(f)=\lim _{n \rightarrow \infty} \psi\left(f_{n}\right)$. By the previous argument $\widetilde{\varphi}$ and $\widetilde{\psi}$ are well defined. It is easy to see that $\tilde{\varphi}, \tilde{\psi} \in K_{\mathbb{R}}(\bar{A})$ and $(1-t) \tilde{\varphi}+t \tilde{\psi}=\operatorname{Re} \varepsilon_{x}$ on $\bar{A}$. On the other hand, $\varepsilon_{x}$ is an extreme point of $K_{\mathbb{R}}(\bar{A})$ since $x \in \operatorname{Ch}(\bar{A}, X)$. Therefore, $\widetilde{\varphi}=\widetilde{\psi}=\varepsilon_{x}$ on $\bar{A}$ and $\operatorname{so} \varphi=\psi=e_{x}$ on $A$. It follows that $e_{x}$ is an extreme point $K_{\mathbb{R}}(A)$ and so $x \in C h(A, X)$. 
(ii) This is proved exactly with the same argument as in part (i) and applying Theorem 8(ii).

(iii) With the same argument as in part (i), by applying Theorem 4(ii), we can show that $\operatorname{Ch}(B, X)=\operatorname{Ch}(\bar{B}, X)$. Therefore, by Theorem 4(iv), we conclude that $\Gamma(B, X)=$ $\Gamma(\bar{B}, X)$.

We now recall the following known result and then extend it by omitting the uniformly closed condition for $A$.

Theorem 10 (see [1, Lemma 3.1 and Theorem 3.2]). Let $A$ be a uniformly closed real subalgebra of $C(X)$ containing 1 and let $\operatorname{Re} A$ separate the points of $X$. Then

(i) A subset $S$ of $X$ is a boundary for $A$ if and only if it is a Choquet set for $A$.

(ii) $C h(A, X)$ is a boundary for $A$.

(iii) The closure of $\operatorname{Ch}(A, X)$ is equal to $\Gamma(A, X)$.

Theorem 11. Let $A$ be a real subalgebra of $C(X)$ containing 1 and let $\operatorname{Re} A$ separate the points of $X$. Then

(i) $\Gamma(A, X)=\Gamma(\bar{A}, X)$.

(ii) $C h(A, X)$ is a boundary for $\bar{A}$ and hence for $A$.

(iii) The closure of $C h(A, X)$ is equal to $\Gamma(A, X)$.

(iv) A closed subset $S$ of $X$ is a boundary for $A$ if and only if it is a Choquet set for $A$.

Proof. Since $\bar{A}$ satisfies the hypotheses of Theorem 10, $\operatorname{Ch}(\bar{A}, X)$ is a boundary for $\bar{A}$ and $\overline{C h(\bar{A}, X)}=\Gamma(\bar{A}, X)$. Moreover, we have $\operatorname{Ch}(A, X)=\operatorname{Ch}(\bar{A}, X)$ by Theorem 9(ii) and $\overline{C h(A, X)} \subseteq \Gamma(A, X)$ by Theorem 5. Therefore, $C h(A, X)$ is a boundary for $\bar{A}$ (hence for $A$ ) and $\Gamma(\bar{A}, X) \subseteq \Gamma(A, X)$. Since $\Gamma(A, X) \subseteq \Gamma(\bar{A}, X)$ it follows that $\Gamma(A, X)=\Gamma(\bar{A}, X)$ and, moreover, $\overline{C h(A, X)}=\Gamma(A, X)$.

To prove (iv) let $S$ be a closed boundary for $A$. By [1, Lemma 1.1], $S$ is a closed Choquet set for $A$. Conversely, if $S$ is a closed Choquet set for $A$, then $\overline{\operatorname{Ch}(A, X)} \subseteq S$ by Theorem 6(iv). Therefore, $S$ is a boundary for $A$ by (ii).

It is interesting to note that in the previous theorem if Re $A$ does not separate the points of $X$, a closed Choquet set may not be a boundary for $A[1$, Lemma 1.1].

The following result is also known and we bring it here for easy reference.

Theorem 12 (see [4, Theorem 4.2.5] and [9]). Let $A$ be a uniformly closed real subalgebra of $C(X, \tau)$ containing 1 and let $\operatorname{Re} A$ separate the points of $X / \tau$. Then

(i) a $\tau$-invariant subset $S$ of $X$ is a boundary for $A$ if and only if it is a Choquet set for $A$.

(ii) $\operatorname{Ch}(A, X, \tau)$ is a $\tau$-invariant boundary for $A$.

(iii) The closure of $\operatorname{Ch}(A, X, \tau)$ is equal to $\Gamma(A, X, \tau)$, the smallest $\tau$-invariant closed boundary for $A$.

We now extend the previous theorem by omitting the uniformly closed condition for $A$.
TABLE 1

\begin{tabular}{ll}
\hline$C(X)$ & $C(X, \tau)$ \\
points of $X$ & points of $X / \tau$ \\
$C h(\bar{A}, X)$ & $C h(\bar{A}, X, \tau)$ \\
(closed) boundary & $\tau$-invariant (closed) boundary \\
$\Gamma(\bar{A}, X)$ & $\Gamma(\bar{A}, X, \tau)$ \\
Theorem 10 & Theorem 12 \\
$C h(A, X)$ & $C h(A, X, \tau)$ \\
$\Gamma(A, X)$ & $\Gamma(A, X, \tau)$ \\
Theorem 5 & Theorem $7($ ii) \\
Choquet set & $\tau$-invariant Choquet set \\
{$[1$, Lemma 1.1] } & Theorem 7(i). \\
\hline
\end{tabular}

Theorem 13. Let $A$ be a real subalgebra of $C(X, \tau)$ containing 1 and let $\operatorname{Re} A$ separate the points of $X / \tau$. Then

(i) $\Gamma(A, X, \tau)=\Gamma(\bar{A}, X, \tau)$.

(ii) $\operatorname{Ch}(A, X, \tau)$ is a $\tau$-invariant boundary for $A$.

(iii) The closure of $\operatorname{Ch}(A, X, \tau)$ is equal to $\Gamma(A, X, \tau)$, the smallest $\tau$-invariant closed boundary for $A$.

(iv) A $\tau$-invariant closed subset $S$ of $X$ is a boundary for $A$ if and only if it is a Choquet set for $A$.

Proof. The proof is similar to that of Theorem 11 by replacing entries in the first column by the corresponding entries in the second column of Table 1.

Corollary 14. If $A$ is a real Banach function algebra on $(X, \tau)$, then

(i) $\operatorname{Ch}(A, X, \tau)=\operatorname{Ch}(\bar{A}, X, \tau)$.

(ii) $\operatorname{Ch}(A, X, \tau)$ is a $\tau$-invariant boundary for $A$.

(iii) $\Gamma(A, X, \tau)=\Gamma(\bar{A}, X, \tau)$.

(iv) $\Gamma(A, X, \tau)=\overline{C h(A, X, \tau)}$.

Proof. Since $A$ separates the points of $X$, we conclude that Re $A$ separates the points of $X / \tau$. Therefore, the result follows by Theorem 9(ii) and Theorem 13.

The following result is also known.

Theorem 15 (see [9, Theorem 3.7, Corollary 3.8] or [4, Theorem 4.3.7]). Let $A$ be a real uniform function algebra on $(X, \tau)$ and suppose that $B=\{f+i g: f, g \in A\}$. Then

$$
C h(A, X, \tau)=C h(B, X), \quad \Gamma(A, X, \tau)=\Gamma(B, X) .
$$

We now extend the previous theorem by omitting the uniformly closed condition for $A$.

Theorem 16. Let $A$ be a real subalgebra of $C(X, \tau)$ containing 1 and separating the points of $X$ and let $B=\{f+i g: f, g \in A\}$. Then $\operatorname{Ch}(A, X, \tau)=\operatorname{Ch}(B, X)$ and $\Gamma(A, X, \tau)=\Gamma(B, X)$. 
Proof. Since $A$ separates the points of $X$, we conclude that Re $A$ separates the points of $X / \tau$. Therefore, $\operatorname{Ch}(A, X, \tau)=$ $\operatorname{Ch}(\bar{A}, X, \tau)$ and $\Gamma(A, X, \tau)=\Gamma(\bar{A}, X, \tau)$ by Theorem 9 (ii) and Theorem 13(i). Clearly, $\bar{A}$ is real uniform function algebra on $(X, \tau)$. Since

$$
\begin{gathered}
B=\{f+i g: f, g \in A\}, \quad C(X)=C(X, \tau) \oplus i C(X, \tau), \\
\max \left\{\|f\|_{X},\|g\|_{X}\right\} \leq\|f+i g\|_{X}
\end{gathered}
$$

for all $f, g \in C(X, \tau)$, we have $\bar{B}=\{f+i g: f, g \in \bar{A}\}$. Hence, $\operatorname{Ch}(\bar{A}, X, \tau)=\operatorname{Ch}(\bar{B}, X)$ and $\Gamma(\bar{A}, X, \tau)=\Gamma(\bar{B}, X)$ by Theorem 15 . On the other hand, since $B$ is a complex subspace of $C(X)$ which contains 1 and separates the points of $X$, we have $\operatorname{Ch}(B, X)=\operatorname{Ch}(\bar{B}, X)$ and $\Gamma(B, X)=\Gamma(\bar{B}, X)$ by Theorem 9(iii). Thus the result follows.

Corollary 17. If $A$ is a real Banach function algebra on $(X, \tau)$ and $B=\{f+i g: f, g \in A\}$, then $\operatorname{Ch}(A, X, \tau)=\operatorname{Ch}(B, X)$ and $\Gamma(A, X, \tau)=\Gamma(B, X)$.

At the end of this section, we present two examples of real subalgebras of $C(X, \tau)$ which are not uniformly closed and then determine their Choquet and Shilov boundaries by Theorem 9, Theorem 13, and Theorem 16.

Example 18. Let $(X, d)$ be a compact metric space and let $0<$ $\alpha \leq 1$. Let $\operatorname{Lip}(X, \alpha)$ be the complex algebra of all complexvalued functions $f$ on $X$ for which

$$
p_{\alpha}(f)=\sup \left\{\frac{|f(x)-f(y)|}{d^{\alpha}(x, y)}: x, y \in X, x \neq y\right\}
$$

is finite. For $0<\alpha<1$, we take $\operatorname{lip}(X, \alpha)$ to be the set of all $f \in \operatorname{Lip}(X, \alpha)$ for which

$$
\frac{|f(x)-f(y)|}{d^{\alpha}(x, y)} \longrightarrow 0 \quad \text { as } d(x, y) \rightarrow 0 .
$$

The Lipschitz norm $\|\cdot\|_{\alpha}$ on $\operatorname{Lip}(X, \alpha)$ is defined by

$$
\|f\|_{\alpha}=\|f\|_{X}+p_{\alpha}(f) \text {. }
$$

It is known that $\left(\operatorname{Lip}(X, \alpha),\|\cdot\|_{\alpha}\right)$ and $\left(\operatorname{lip}(X, \alpha),\|\cdot\|_{\alpha}\right)$ are complex Banach function algebras on $X$. These algebras are called Lipschitz algebras of order $\alpha$ and were first studied by Sherbert in $[13,14]$.

Let $\tau$ be a Lipschitz involution on $(X, d)$, that is, an involution $\tau$ on $X$ satisfying the Lipschitz condition $d(\tau(x), \tau(y)) \leq$ $C d(x, y)$ for a positive constant $C$ and for all $x, y$ in $X$. Let $\sigma$ be the algebra involution induced by $\tau$ on $C(X)$. Then $\sigma(\operatorname{Lip}(X, \alpha))=\operatorname{Lip}(X, \alpha)$ and $\sigma(\operatorname{lip}(X, \alpha))=\operatorname{lip}(X, \alpha)$; that is, these (complex) Lipschitz algebras are $\sigma$-invariant. We now define

$$
\begin{aligned}
\operatorname{Lip}(X, \tau, \alpha) & =\{h \in \operatorname{Lip}(X, \alpha): \sigma(h)=h\}, \\
\operatorname{lip}(X, \tau, \alpha) & =\{h \in \operatorname{lip}(X, \alpha): \sigma(h)=h\} .
\end{aligned}
$$

Then $\operatorname{Lip}(X, \tau, \alpha)$ and $\operatorname{lip}(X, \tau, \alpha)$ are real Banach function algebras on $(X, \tau)$ under the norm $\|\cdot\|_{\alpha}$ and we have

$$
\begin{gathered}
\operatorname{Lip}(X, \alpha)=\operatorname{Lip}(X, \tau, \alpha) \oplus i \operatorname{Lip}(X, \tau, \alpha), \\
\operatorname{lip}(X, \alpha)=\operatorname{lip}(X, \tau, \alpha) \oplus i \operatorname{lip}(X, \tau, \alpha) .
\end{gathered}
$$

These algebras are called real Lipschitz algebras of complex functions of order $\alpha$ and were first studied in [15].

Since $\operatorname{Lip}(X, 1) \subseteq \operatorname{lip}(X, \alpha) \subseteq \operatorname{Lip}(X, \alpha)$ and for each $x \in$ $X$ the function $f_{x}: X \rightarrow \mathbb{C}$, defined by

$$
f_{x}(y)=1-\frac{d(x, y)}{\operatorname{diam} X}
$$

belongs to $\operatorname{Lip}(X, 1)$ and peaks at $x$, we conclude that

$$
\begin{aligned}
S_{0} & (\operatorname{Lip}(X, 1), X) \\
& =S_{0}(\operatorname{lip}(X, \alpha), X)=S_{0}(\operatorname{Lip}(X, \alpha), X)=X .
\end{aligned}
$$

If $B$ is the algebra $\operatorname{Lip}(X, \alpha)$ for $\alpha \in(0,1]$ or $\operatorname{lip}(X, \alpha)$ for $\alpha \in(0,1)$, then by Theorem $16, C h(B, X)=C h(A, X, \tau)$ and $\Gamma(A, X, \tau)=\Gamma(B, X)$, whenever $A=\operatorname{Lip}(X, \tau, \alpha)$, for $\alpha \in(0,1]$, or $A=\operatorname{lip}(X, \tau, \alpha)$, for $\alpha \in(0,1)$. Since $\bar{B}=C(X)$ and

$$
\operatorname{Ch}(B, X)=\operatorname{Ch}(\bar{B}, X)=\Gamma(B, X)=\Gamma(\bar{B}, X)=X,
$$

we have $C h(A, X, \tau)=\Gamma(A, X, \tau)=X$.

Example 19. Let $X$ be the closed unit interval $[0,1]$ with the usual Euclidean topology. Let $B=D^{\infty}(X)$ be the complex algebra of all (continuous) complex-valued functions with derivatives of all orders on $X$. It is known that $B$ is not complete under any algebra norm. For this property see, for example, Carpenter's theorem [16] or Singer-Wermer theorem $[17, \S 18$, Theorem 16]. Since $B$ is a self-adjoint complex subalgebra of $C(X)$ which contains 1 and separates the points of $X$, we have $\bar{B}=C(X)$ by the Stone-Weierstrass theorem for complex subalgebras of $C(X)$. Since $C h(C(X), X)=$ $\Gamma(C(X), X)=X$, by Theorem 9 (iii) it follows that $C h(B, X)=$ $\Gamma(B, X)=X$.

We now define the topological involution $\tau$ on $X$ by $\tau(x)=1-x$ and let $\sigma$ be the algebra involution induced by $\tau$ on $C(X)$. Since for every $h \in B, \sigma(h)$ is a differentiable function of all orders on $X$ and $(\sigma(h))^{(n)}=(-1)^{n} \sigma\left(h^{(n)}\right)$ for each $n \in \mathbb{N}$, it follows that $\sigma(B)=B$. If we define $A=$ $\{h \in B: \sigma(h)=h\}$, then $A$ is a real subalgebra of $C(X, \tau)$ containing 1 , separating the points of $X$, and moreover, the algebra $B=\{f+i g: f, g \in A\}$ is the complexification of $A$. Then by [15, Theorem 1.2], $A$ is not complete under any algebra norm. Since $C h(B, X)=\Gamma(B, X)=X$, we conclude that $C h(A, X, \tau)=\Gamma(A, X, \tau)=X$ by Theorem 16 .

On the other hand, $\bar{A}=C(X, \tau)$ by the StoneWeierstrass theorem for real subalgebras of $C(X, \tau)$ [3]. Since $C h(C(X, \tau), X)=\Gamma(C(X, \tau), X)=X$, we conclude that $\operatorname{Ch}(A, X, \tau)=\Gamma(A, X, \tau)=X$ by Theorem 9 (ii) and Theorem 13(i). 


\section{Peak Sets and Peak Points}

In this section we extend some of the known results concerning peak sets and $p$-sets for complex Banach (uniform, resp.) function algebras to those for real Banach (uniform, resp.) function algebras.

We know that every complex uniform function algebra on $X$ can be regarded as a real uniform function algebra on a compact Hausdorff space with a suitable topological involution $\tau$ [3]. We may take $Y$ as a disjoint union of two copies of $X$ and $\tau$ as a homeomorphism that sends a point in one copy of $X$ to the corresponding point in the other copy of $X$ [4, page 29]. Now a natural question arises here whether the above fact holds for Banach function algebras. The answer is affirmative as the following result shows. However, its proof is similar to that of Kulkarni and Limaye for uniform function algebras.

Theorem 20. Let $(B,|||\cdot|||)$ be a complex Banach function algebra on a compact Hausdorff space X. Then there exists a compact Hausdorff space $Y$, a topological involution $\tau$ on $Y$, and a real Banach function algebra $(A,\|\cdot\|)$ on $(Y, \tau)$ such that $(B,|||\cdot|||)$, regarded as a real Banach algebra, is isometrically isomorphic to $(A,\|\cdot\|)$. Moreover,

(i) the algebra $A$ is natural if and only if $B$ is natural.

(ii) There exists a one-to-one correspondence between the set of all peak sets ( $p$-sets, resp.) for $B$ and the set of all peak sets ( $p$-sets, resp.) for $A$, with respect to $(Y, \tau)$.

Proof. Let $Y$ be the compact Hausdorff space $X \times\{0,1\}$ with the product topology. We define the map $\tau: Y \rightarrow Y$ by $\tau(x, 0)=(x, 1)$ and $\tau(x, 1)=(x, 0)$ for all $x \in X$. Clearly, $\tau$ is a topological involution on $Y$. Now, we define the map $\Psi: C(X) \rightarrow C(Y, \tau)$ by

$$
\begin{gathered}
\Psi(f)(x, 0)=f(x), \\
\Psi(f)(x, 1)=\overline{f(x)} \quad(f \in C(X), x \in X) .
\end{gathered}
$$

Then $\Psi$ is an isometrically isomorphism from $\left(C(X),\|\cdot\|_{X}\right)$, regarded as a real Banach algebra, onto $\left(C(Y),\|\cdot\|_{Y}\right)$ and $\Psi(1)=1$. Set $A=\Psi(B)$. It follows that $A$ is a real subalgebra of $C(Y, \tau)$ which contains 1 and separates the points of $Y$. We define $\|g\|=\left\|\left|\Psi^{-1}(g) \|\right|\right.$ for all $g \in A$. Then $\| \cdot \|$ is a complete algebra norm on $A$ and $\|1\|=1$. Therefore, $A$ is a real Banach function algebra on $(Y, \tau)$. Now, we define the map $\Phi: B \rightarrow$ $A$ by $\Phi=\left.\Psi\right|_{B}$. Clearly, $\Phi$ is an isometric isomorphism from $(B,|||\cdot|||)$, regarded as a real Banach algebra, onto $(A,\|\cdot\|)$.

Let $B_{r}$ denote $B$ as a real algebra. Then it is easy to show that $M_{B_{r}}=M_{B} \cup\left\{\bar{\eta}: \eta \in M_{B}\right\}$. To prove the naturality of $A$, let $\varphi \in M_{A}$. Then $\varphi \circ \Phi \in M_{B_{r}}$. If $B$ is natural, either there exists $x \in X$ such that $\varphi \circ \Phi=e_{x}$ or there exists $y \in X$ such that $\varphi \circ$ $\Phi=\overline{e_{y}}$. We can easily show that $\varphi$ is the evaluation character at $(x, 0)$ or at $(y, 1)$, respectively. Therefore, $A$ is natural.

Conversely, let $A$ be natural and $\eta \in M_{B}$. Hence $\eta \circ \Phi^{-1} \in$ $M_{A}$. Therefore, either there exists $x \in X$ such that $\eta \circ \Phi^{-1}$ is the evaluation character at some $(x, 0)$ on $A$ or it is the evaluation character at some $(y, 1)$ on $A$. Since $\eta \in M_{B}$ and $B$ is a complex algebra, the latter case does not occur, and hence $\eta \circ \Phi^{-1}=e_{(x, 0)}$. Since $\Phi(B)=A$, it follows that $\eta=e_{x}$ and thus $B$ is natural.

Now let $S$ be a peak set ( $p$-set, resp.) for $B$. It is easy to see that $S \times\{0,1\}$ is a peak set ( $p$-set, resp.) for $A$.

Conversely, let $E$ be a peak set for $A$. Then there exists a subset $S$ of $X$ such that $E=S \times\{0,1\}$. We can easily show that $S$ is a peak set for $B$.

If $P$ is a $p$-set for $B$, then $P=\cap_{\alpha \in I} S_{\alpha}$, where $S_{\alpha}$ is a peak set for $B$. Since $P \times\{0,1\}=\cap_{\alpha \in I}\left(S_{\alpha} \times\{0,1\}\right)$, it follows that $P \times\{0,1\}$ is a $p$-set for $A$.

Concerning the union of peak sets and $p$-sets for real or complex subspaces of $C(X)$, the following results are wellknown.

Theorem 21 (see [4, Remark 2.2.10]). Let A be a uniformly closed real subalgebra of $C(X)$ containing 1 . If $S$ and $T$ are peak sets ( $p$-sets, resp.) for $A$, then $S \cup T$ is a peak set ( $p$-set, resp.) for $A$.

Theorem 22 (see [18, II. Corollary 12.8]). Let B be a complex uniform function algebra on $X$. If $\left\{S_{j}\right\}_{j=1}^{\infty}$ is a sequence of $p$-sets for $B$ and $S=\bigcup_{j=1}^{\infty} S_{j}$ is closed, then $S$ is a p-set for $B$.

Note that the above two theorems do not hold for complex Banach function algebras, in general. In fact, Dales has proved the following result in [12] and has shown, by counterexamples, that the restriction imposed on $B$ and the peak sets ( $p$-sets, resp.) are necessary.

Theorem 23. Let $B$ be a natural complex Banach function algebra on $X$. Then a finite union of pairwise disjoint peak sets ( $p$-sets, resp.) for $B$ is a peak set ( $p$-set, resp.) for $B$.

The following two results state the relation between the peak sets ( $p$-sets, resp.) for a real subspace of $C(X, \tau)$ and those of its complexification, which are modifications of [4, Theorem 2.2.11].

Theorem 24. Let $A$ be a real subspace of $C(X, \tau)$ containing 1 and $B=\{f+i g: f, g \in A\}$.

(i) If $S$ is a peak set (p-set, resp.) for $A$, then it is a peak set (p-set, resp.) for B. Moreover, $\tau(S)$ is also a peak set (p-set, resp.) for $A$ and $\tau(S)=S$.

(ii) If $S$ is a peak set (p-set, resp.) for $B$, then $\tau(S)$ is also a peak set (p-set, resp.) for $B$. In case $\tau(S)=S, S$ is, in fact, a peak set ( $p$-sets, resp.) for $A$.

(iii) $S_{0}(A, X)=S_{0}(B, X) \cap \operatorname{Fix}(\tau)$ and $S(A, X)=S(B, X) \cap$ $\operatorname{Fix}(\tau)$.

Theorem 25. Let $A$ be a uniformly closed subalgebra of $C(X, \tau)$ containing 1 and let $B=\{f+i g: f, g \in A\}$.

(i) If $S$ is a peak set (p-set, resp.) for $B$, then $S \cup \tau(S)$ is a peak set (p-set, resp.) for $A$.

(ii) $S_{0}(B, X) \subseteq T_{0}(A, X, \tau)$ and $S(B, X) \subseteq T(A, X, \tau)$.

(iii) $T_{0}(A, X, \tau) \cap \operatorname{Fix}(\tau) \subseteq S_{0}(B, X)$ and $T(A, X, \tau) \cap$ $\operatorname{Fix}(\tau) \subseteq S_{0}(B, X)$. 
(iv) If $A$ is a real uniform function algebra on $(X, \tau)$, then $T_{0}(A, X, \tau)=S_{0}(B, X)$ and $T(A, X, \tau)=S(B, X)$.

We now extend Theorem 23 for natural real Banach function algebras.

Theorem 26. Let $(A,\|\cdot\|)$ be a natural real Banach function algebra on $(X, \tau)$. Then a finite union of pairwise disjoint peak sets ( $p$-sets, resp.) for $A$ is a peak set ( $p$-set, resp.) for A.

Proof. It is sufficient to show that if $S_{1}$ and $S_{2}$ are two disjoint peak sets ( $p$-sets, resp.) for $A$, then $S_{1} \cup S_{2}$ is a peak set ( $p$-set, resp.) for $A$. Let $B=\{f+i g: f, g \in A\}$. Then $B$ is a complex subalgebra of $C(X)$ and there exists an algebra norm $\mid\|\cdot\| \cdot \|$ on $B$ such that $(B, \||\cdot|||)$ is a natural complex Banach function algebra on $X$ by [15, Theorem 1.2]. On the other hand, $S_{1}$ and $S_{2}$ are peak sets ( $p$-sets, resp.) for $B$ by Theorem 24(i). Thus $S_{1} \cup S_{2}$ is a peak set ( $p$-set, resp.) for $B$ by Theorem 23. Since $S_{1}$ and $S_{2}$ are $\tau$-invariant, $S_{1} \cup S_{2}$ is also $\tau$-invariant. Therefore, $S_{1} \cup S_{2}$ is a peak set ( $p$-set, resp.) for $A$ by Theorem 24(ii).

Remark 27. By using the counter examples in [7] and considering Theorem 20, we can show that all restrictions imposed on $A$ in the above theorem are necessary.

Now, we show that part (i) of Theorem 25 holds for real Banach function algebras with small modification.

Theorem 28. Let $(A,\|\cdot\|)$ be a real Banach function algebra on $(X, \tau)$ and $B=\{f+i g: f, g \in A\}$ be its complexification. If $S$ is a peak set ( $p$-set, resp.) for $B$ then $S \cup \tau(S)$ is a peak set (p-set, resp.) for $\bar{A}$.

Proof. Since $S$ is a peak set ( $p$-set, resp.) for $B$, we conclude that $\tau(S)$ is also a peak set ( $p$-set, resp.) for $B$ by Theorem 24(ii). Let $D=\{f+i g: f, g \in \bar{A}\}$ be the complexification of $\bar{A}$. Clearly, $S$ and $\tau(S)$ are peak sets ( $p$ sets, resp.) for $D$ and hence by Theorem 25(i), $S \cup \tau(S)$ is a peak set ( $p$-set, resp.) for $\bar{A}$.

Theorem 29. Let $(B,\|\cdot\|)$ be a natural complex Banach function algebra on $X$ and $\sigma$ be the algebra involution on $C(X)$, induced by the topological involution $\tau$. Let $\sigma(B)=B$ and $A=\{h \in B: \sigma(h)=h\}$. If $S_{1}$ and $S_{2}$ are $\tau$-invariant peak sets ( $p$-sets, resp.) for $B$ such that $S_{1} \cap S_{2}=\phi$, then $S_{1} \cup S_{2}$ is a peak set ( $p$-set, resp.) for $A$.

Proof. By [15, Theorem 1.1], $(A,\|\cdot\|)$ is a natural real Banach function algebra on $(X, \tau)$ and $B=\{f+i g: f, g \in A\}$. On the other hand, $S_{1}$ and $S_{2}$ are peak sets ( $p$-sets, resp.) for $A$ by Theorem 24(ii). Therefore, $S_{1} \cup S_{2}$ is a peak set( $p$-set, resp.) for $A$ by Theorem 26.

The following example shows that the uniformly closed condition of $A$ in Theorem 25(i) and the condition $S_{1} \cap S_{2}=\emptyset$ in Theorem 29 are essential.

Example 30. Let $X=\left\{(z, w) \in \mathbb{C}^{2}:|z| \leq 1,|w| \leq 1\right\}$ and let $B$ be the complex algebra of all continuous complex-valued functions on $X$ which have continuous first-order partial derivatives with respect to $z$ and $w$. We define the algebra norm $\|\cdot\|$ on $B$ by

$$
\|f\|=\|f\|_{X}+\left\|\frac{\partial f}{\partial z}\right\|_{X}+\left\|\frac{\partial f}{\partial w}\right\|_{X} \quad(f \in B) .
$$

Then $(B,\|\cdot\|)$ is a natural complex Banach function algebra on $X$ and although the sets $S_{1}=\{(1, w):|w| \leq 1\}$ and $S_{2}=$ $\{(z, 1):|z| \leq 1\}$ are peak sets for $B$, the set $S_{1} \cup S_{2}$ is not a peak set for $B$, as shown by Dales in [12, Example 1]. Note that $S_{1} \cap S_{2}$ is nonempty.

We first define the topological involution $\tau$ on $X$ by $\tau(z, w)=(\bar{w}, \bar{z})$. Let $\sigma$ be the algebra involution on $C(X)$, induced by $\tau$. By the definition of $B$, for every $f \in B$ and for each fixed $w$ in the closed unit disk $\overline{\mathbb{D}}, f(\bar{w}, z)$ has continuous derivative on $\overline{\mathbb{D}}$ and hence $\bar{f}(\bar{w}, \bar{z})$ has also continuous derivative on $\overline{\mathbb{D}}$ as a function of $z$. Similarly, for each fixed $z \in \mathbb{\mathbb { D }}, f(w, \bar{z})$ has continuous derivative on $\overline{\mathbb{D}}$ and hence, as a function of $w, \bar{f}(\bar{w}, \bar{z})$ has also continuous derivative on $\overline{\mathbb{D}}$. Therefore, $\bar{f}(\bar{w}, \bar{z})$ has continuous partial derivatives on $X$ with respect to $z$ and $w$. Since $\bar{f}(\bar{w}, \bar{z})=$ $\bar{f} \circ \tau(z, w)$, for all $(z, w) \in X$, it follows that $\sigma(f)=\bar{f} \circ \tau \in B$ and hence $\sigma(B)=B$. If we define $A=\{h \in B: \sigma(h)=h\}$, then $(A,\|\cdot\|)$ is a natural real Banach function algebra on $(X, \tau)$ and $B=\{f+i g: f, g \in A\}$ is the complexification of $A$ by [15, Theorem 1.1]. Moreover, $A$ is not uniformly closed. Since $\tau\left(S_{1}\right)=S_{2}$, the set $S_{1} \cup \tau\left(S_{1}\right)$ is not a peak set for $B$. Therefore, $S_{1} \cup \tau\left(S_{1}\right)$ is not a peak set for $A$ by Theorem 24(i).

We now define the topological involution $\tau$ on $X$ by $\tau(z, w)=(\bar{z}, \bar{w})$. Clearly, $S_{1}$ and $S_{2}$ are $\tau$-invariant. Let $\sigma$ be the algebra involution on $C(X)$, induced by $\tau$. By a similar argument as in the above case, it is easy to see that $\sigma(B)=B$. If we define $A=\{h \in B: \sigma(h)=h\}$, then $A$ is a natural real Banach function algebra on $(X, \tau)$ and $B=\{f+i g: f, g \in A\}$ by [15, Theorem 1.1]. Since $S_{1} \cup S_{2}$ is not a peak set for $B$, it is not a peak set for $A$ by Theorem 24(i).

Theorem 31. Let $A$ be a natural real Banach function algebra on $(X, \tau)$ and let $B=\{f+i g: f, g \in A\}$. Then $S_{0}(B, X) \subseteq$ $T_{0}(A, X, \tau)$ and $S(B, X) \subseteq T(A, X, \tau)$.

Proof. Let $x \in S_{0}(B, X)$. If $x=\tau(x)$ then $x_{0} \in S_{0}(A, X)$ by Theorem 24(ii), and hence $x \in T_{0}(A, X, \tau)$. If $x \neq \tau(x)$, then $\{x, \tau(x)\}$ is a peak set for $B$ by Theorem 24(ii) and Theorem 23. Since $\{x, \tau(x)\}$ is $\tau$-invariant, it follows that it is a peak set for $A$ by Theorem 24(ii) and hence $x \in T_{0}(A, X, \tau)$. Therefore, the first inclusion holds.

By applying parts (i) and (iii) in Theorem 24 and Theorem 29 for $p$-sets, the second inclusion holds with the same argument.

Concerning the existence of a $p$-point (a peak point, respectively) in every peak set for a complex uniform function algebra, the following result holds.

Theorem 32 (see [2, Corollary 2.4.6]). Let B a complex uniform function algebra on $X$. Then every peak set for $B$ 
contains a p-point for B. Moreover, if $X$ is first countable, then every peak set for $B$ contains a peak point for $B$.

Note that the above theorem is not true for complex Banach function algebras, in general (see $[11,19,20])$.

The following example shows that the above theorem does not hold even for real uniform function algebras, in general.

Example 33. We denote by $\mathbb{D}, \overline{\mathbb{D}}$, and $\mathbb{\mathbb { T }}$ the open unit disk $\{z \in \mathbb{C}:|z|<1\}$, the closed unit $\operatorname{disc}\{z \in \mathbb{C}:|z| \leq 1\}$, and the unit circle $\{z \in \mathbb{C}:|z|=1\}$, respectively. Let $B=$ $A(\overline{\mathbb{D}})$, the complex disc algebra. It is known that $\left(B,\|\cdot\|_{\overline{\mathbb{D}}}\right)$ is a complex uniform algebra on $\overline{\mathbb{D}}$ and $S_{0}(B ; \overline{\mathbb{D}})=\mathbb{T}$. We define the topological involution $\tau$ on $\overline{\mathbb{D}}$ by $\tau(z)=\bar{z}$. Let $A=\{h \in$ $B: \sigma(h)=h$, where $\sigma$ is the algebra involution induced by $\tau$ on $C(\overline{\mathbb{D}})$. Since $\sigma(f)(z)=\bar{f}(\bar{z})$ is continuous on $\overline{\mathbb{D}}$ and it is analytic on $\mathbb{D}$ for each $f \in B$, it follows that $\sigma(B)=B$. In fact, $A$ is the real disc algebra and $B=\{f+i g: f, g \in A\}$ is the complexification of $A$. Moreover,

$$
S_{0}(A, \overline{\mathbb{D}})=S_{0}(B, \overline{\mathbb{D}}) \cap \operatorname{Fix}(\tau)=\mathbb{T} \cap\{-1,1\}=\{-1,1\},
$$

by Theorem 24(iii). Hence for each $z \in \mathbb{T}$ with $\bar{z} \neq z$, the set $\{z, \bar{z}\}$ is a peak set for $A$ which does not contain any peak point for $A$.

The following is a modification of Theorem 32 for real uniform function algebras.

Theorem 34. Let $A$ be a real uniform function algebra on $(X, \tau)$. Then every peak set for $A$ contains a $\tau$-p-point for $A$. Moreover, if $X$ is first countable, every peak set for A contains a $\tau$-peak point for $A$.

Proof. Let $S$ be a peak set for $A$. If we take $B=\{f+i g$ : $f, g \in A\}$ then $B$ is a complex uniform function algebra on $X$ by [4, Theorem 1.3.20] and $S$ is also a peak set for $B$. Hence $S \cap S(B, X) \neq \emptyset$ and when $X$ is first countable, $S \cap$ $S_{0}(B, X) \neq \emptyset$, by Theorem 32. Since $S(B, X)=T(A, X, \tau)$ and $S_{0}(B, X)=T_{0}(A, X, \tau)$, we have $S \cap T(A, X, \tau) \neq \emptyset$, and if $X$ is first countable, then $S \cap T_{0}(A, X, \tau) \neq \emptyset$.

\section{On the Density of $\tau$-Peak Points in the Shilov Boundary}

We first state some known results on the density of the set of peak points in the Shilov boundary for complex Banach function algebras.

Theorem 35 (see [2, Theorem 2.3.4]). Let $B$ be a complex uniform function algebra on $X$. Then $S(B, X)=C h(B, X)$. Moreover, if $X$ is first countable, then $S_{0}(B, X)=C h(B, X)$ and so $\Gamma(B, X)$ is the closure of $S_{0}(B, X)$.

Theorem 36 (see [11, Theorem 2.3] and [21]). Let $B$ be a Banach function algebra on a compact metrizable space $X$. Then $\Gamma(B, X)$ is the closure of $S_{0}(B, X)$.
The following example shows that the above theorem does not hold even for real uniform algebras on compact metrizable spaces, in general.

Example 37. Let $B$ be the complex disc algebra and let $A$ be the real disc algebra, considered in Example 33. It is known that $S_{0}(B, \overline{\mathbb{D}})=\Gamma(B ; \overline{\mathbb{D}})=\mathbb{T}$. Therefore, $\Gamma(A, \overline{\mathbb{D}}, \tau)=\mathbb{T}$ by Theorem 16. On the other hand, $S_{0}(A, \overline{\mathbb{D}})=\{-1,1\}$ as shown in Example 33. Hence, the closure of $S_{0}(A, \overline{\mathbb{D}})$ is not equal to $\Gamma(A, X, \tau)$.

Theorem 38 (see [10] or [4, Theorem 4.2.4]). Let $A$ be a uniformly closed real subalgebra of $C(X, \tau)$ containing 1 . Then

$$
C h(A, X, \tau)=T(A, X, \tau) .
$$

Remark 39. Let $A$ be a real uniform function algebra on $(X, \tau)$ and $B=\{f+i g: f, g \in A\}$. Then we have $C h(A, X, \tau)=T(A, X, \tau)$ by Theorem 38, $C h(A, X, \tau)=$ $C h(B, X)$ by Theorem 15, and $C h(B, X)=S(B, X)$ by Theorem 35. Therefore, $T(A, X, \tau)=S(B, X)$, which has already been obtained in Theorem 25(iv).

Theorem 40. Let $A$ be a uniformly closed real subalgebra of $C(X, \tau)$ containing 1 and let $\operatorname{Re} A$ separate the points of $X / \tau$. Then

$$
\overline{T(A, X, \tau)}=\Gamma(A, X, \tau)
$$

In particular, if $X$ is a first countable space, then

$$
\overline{T_{0}(A, X, \tau)}=\Gamma(A, X, \tau) .
$$

Proof. By Theorem 38 we have $T(A, X, \tau)=C h(A, X, \tau)$ and since $\overline{C h(A, X, \tau)}=\Gamma(A, X, \tau)$ by Theorem 13(iii), we conclude that

$$
\overline{T(A, X, \tau)}=\Gamma(A, X, \tau)
$$

In the case that $X$ is first countable, every finite subset of $X$ is a $G_{\delta}$-set. Hence $T(A, X, \tau) \subseteq T_{0}(A, X, \tau)$ and so $T(A, X, \tau)=T_{0}(A, X, \tau)$. Therefore, $\overline{T_{0}(A, X, \tau)}=\Gamma(A, X, \tau)$ by the previous argument.

Theorem 41. Let $X$ be a compact metrizable space and let $\tau$ be a topological involution on $X$. If $(A,\|\cdot\|)$ is a natural real Banach function algebra on $(X, \tau)$, then

$$
\overline{T_{0}(A, X, \tau)}=\Gamma(A, X, \tau) .
$$

Proof. Let $B=\{f+i g: f, g \in A\}$. The naturality of $A$ implies that $S_{0}(B, X) \subseteq T_{0}(A, X, \tau)$ by Theorem 31. On the other hand, there exists an algebra norm ||$|\cdot|||$ on $B$ such that $(B,|||\cdot|||)$ is a complex Banach function algebra on $X$ by [15, Theorem 1.2]. Since $X$ is a metrizable compact space, we have $\overline{S_{0}(B, X)}=\Gamma(B, X)$ by Theorem 36. On the other hand, $\Gamma(A, X, \tau)=\Gamma(B, X)$ by Theorem 16. Therefore, $\overline{S_{0}(B, X)}=$ $\overline{T_{0}(A, X, \tau)}=\Gamma(A, X, \tau)$. 


\section{References}

[1] S. H. Kulkarni and B. V. Limaye, "Boundaries and Choquet sets for real subspaces of $C(X)$," Mathematica Japonica, vol. 51, no. 2, pp. 199-212, 2000.

[2] A. Browder, Intruduction to Function Algebras, W. A. Benjamin, New York, NY, USA, 1969.

[3] S. H. Kulkarni and B. V. Limaye, "Gleason parts of real function algebras," Canadian Journal of Mathematics, vol. 33, no. 1, pp. 181-200, 1981.

[4] S. H. Kulkarni and B. V. Limaye, Real Function Algebras, Marcel Dekker, New York, NY, USA, 1992.

[5] W. Rudin, Functional Analysis, McGraw-Hill, New York, NY, USA, 2nd edition, 1991.

[6] L. Ingelstam, "Symmetry in real Banach algebras," Mathematica Scandinavica, vol. 18, pp. 53-68, 1966.

[7] B. V. Limaye, "Boundaries for real Banach algebras," Canadian Journal of Mathematics, vol. 28, no. 1, pp. 42-49, 1976.

[8] B. V. Limaye and R. R. Simha, "Deficiencies of certain real uniform algebras," Canadian Journal of Mathematics, vol. 27, pp. 121-132, 1975.

[9] S. H. Kulkarni and S. Arundhathi, "Choquet boundary for real function algebras," Canadian Journal of Mathematics, vol. 40, no. 5, pp. 1084-1104, 1988.

[10] S. Arundhathi, "A note on the peak points for real function algebras," Indian Journal of Pure and Applied Mathematics, vol. 21, no. 2, pp. 155-162, 1990.

[11] H. G. Dales, "Boundaries and peak points for Banach function algebras," Proceedings of the London Mathematical Society, vol. 22, pp. 121-136, 1971.

[12] H. G. Dales, "The union of peak sets for Banach function algebras," The Bulletin of the London Mathematical Society, vol. 3, pp. 204-208, 1971.

[13] D. R. Sherbert, "Banach algebras of Lipschitz functions," Pacific Journal of Mathematics, vol. 13, pp. 1387-1399, 1963.

[14] D. R. Sherbert, "The structure of ideals and point derivations in Banach algebras of Lipschitz functions," Transactions of the American Mathematical Society, vol. 111, pp. 240-272, 1964.

[15] D. Alimohammadi and A. Ebadian, "Hedberg's theorem in real Lipschitz algebras," Indian Journal of Pure and Applied Mathematics, vol. 32, no. 10, pp. 1479-1493, 2001.

[16] R. L. Carpenter, "Continuity of derivations in F-algebras," American Journal of Mathematics, vol. 93, pp. 500-502, 1971.

[17] F. F. Bonsall and J. Duncan, Complete Normed Algebras, Springer, New York, NY, USA, 1973.

[18] T. W. Gamelin, Uniform Algebras, Chelsea Publishing Company, 1984.

[19] T. G. Honary, "An example of a Banach function algebra having a peak set without any peak point," in Proceedings of the 5th Analysis Seminar, pp. 26-33, Shiraz University, 1990.

[20] K. Jarosz, "Peak set without peak points," Proceedings of the American Mathematical Society, vol. 125, no. 5, pp. 1377-1379, 1997.

[21] T. G. Honary, "The density of peak points in the Shilov boundary of a Banach function algebra," Proceedings of the American Mathematical Society, vol. 103, no. 2, pp. 480-482, 1988. 


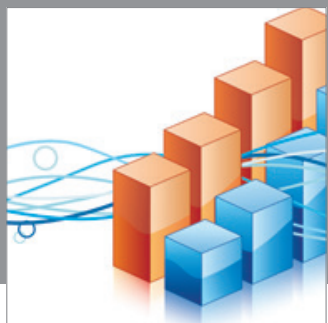

Advances in

Operations Research

mansans

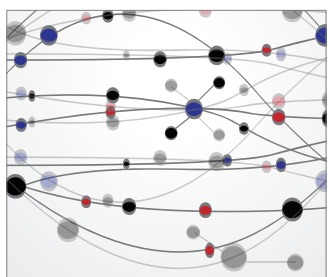

The Scientific World Journal
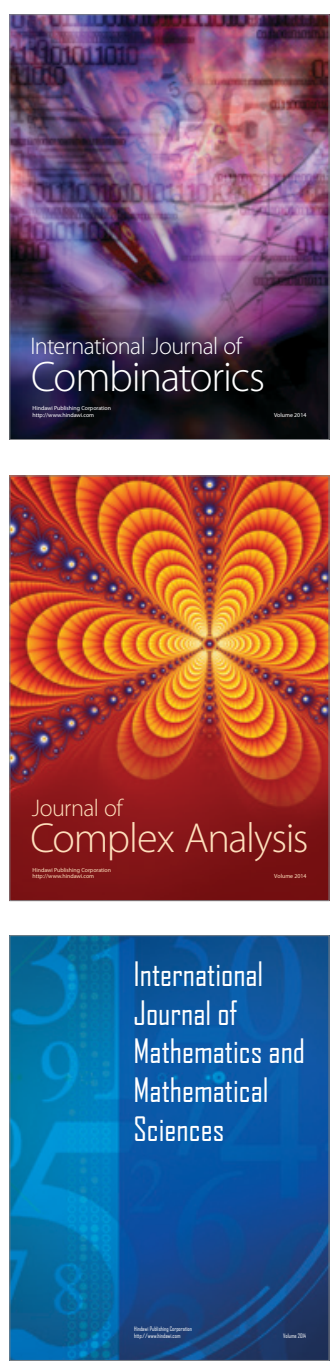
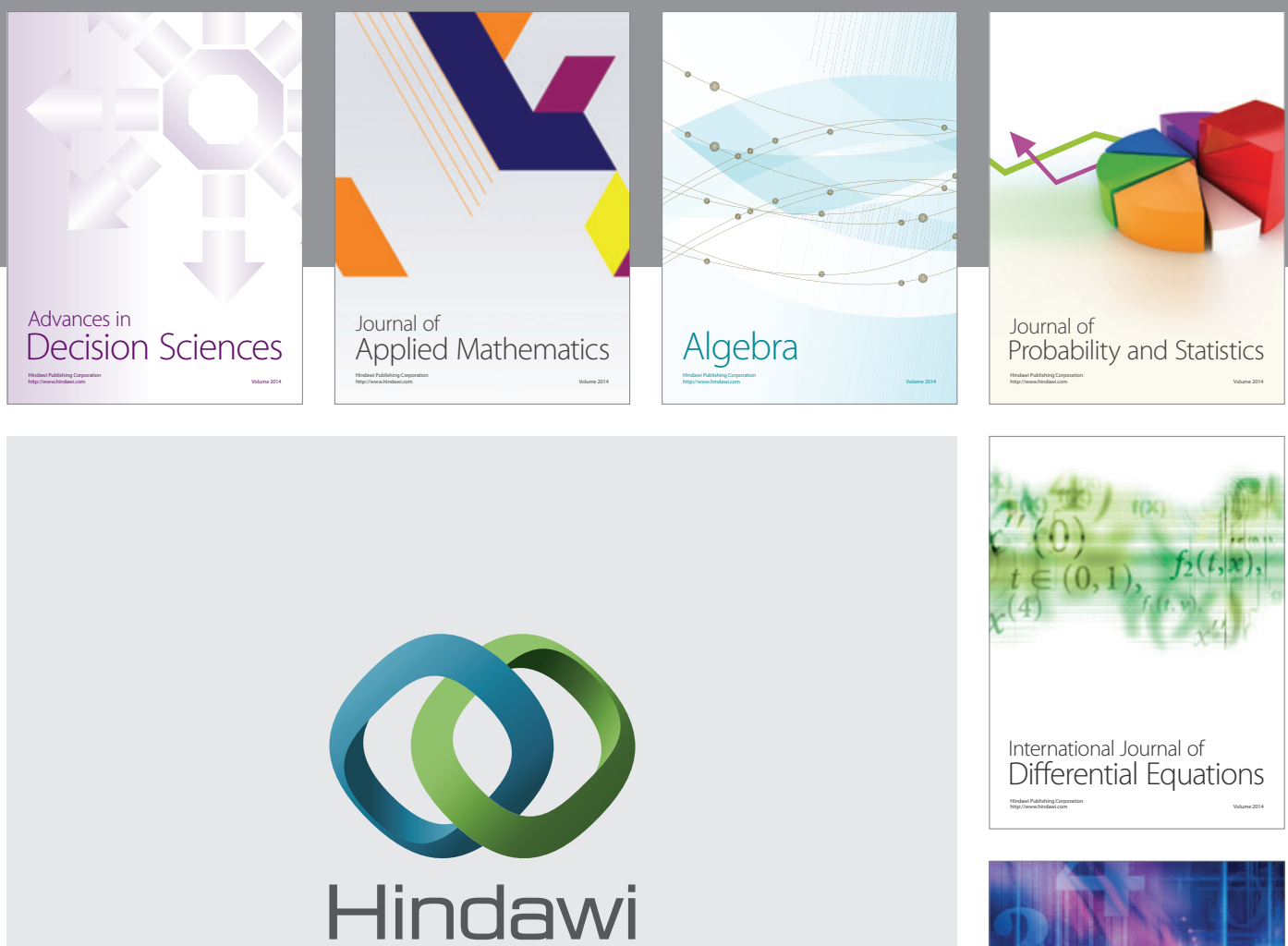

Submit your manuscripts at http://www.hindawi.com
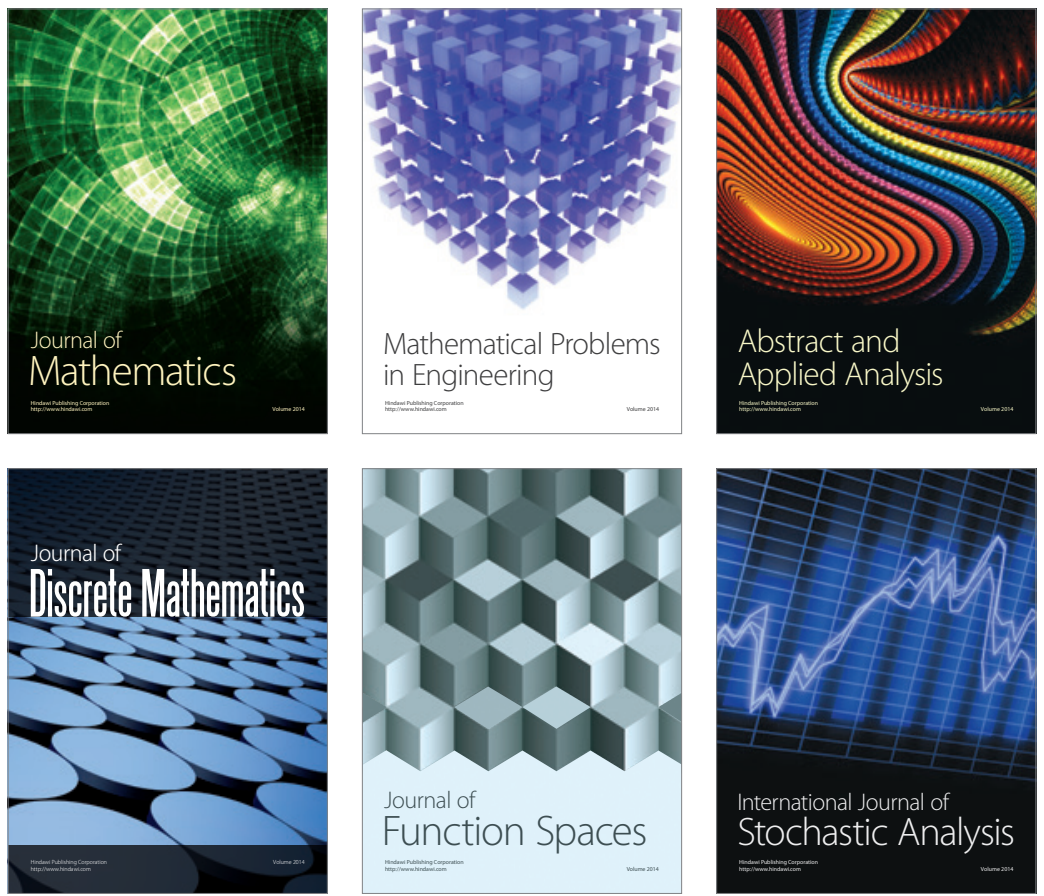

Journal of

Function Spaces

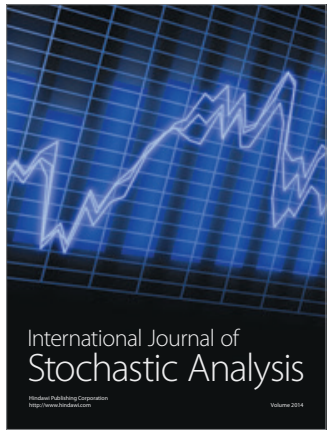

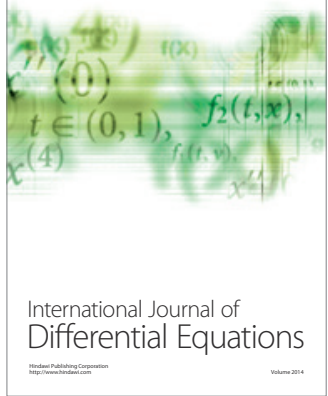
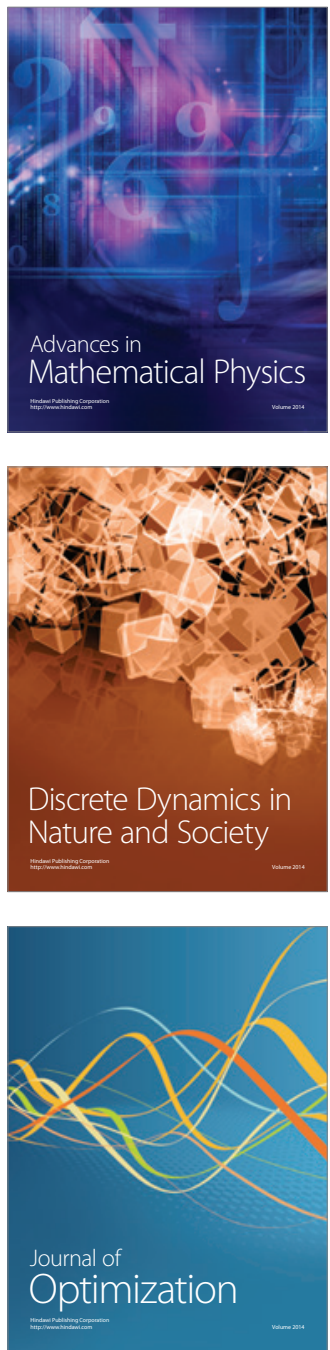